of Medicine report. Arch Gen Psychiatry 57: 547-552.

Wegener, N., Kuhnert, S., Thüns, A., Roese, R. und Koch, M. (2008): Effects of acute systemic and intra-cerebral stimulation of cannabinoid receptors on sensorimotor gating, locomotion, and spatial memory in rats. Psychopharmacology 198: 375-385.

Eine vollständige Literaturliste kann bei den Autoren angefordert werden.

\section{Danksagung}

Die Autoren danken Nina Ptak für die Illustration der Vorgänge rund um den CB-Rezeptor.

\section{Kurzbiografien}

Nico Wegener: geboren 1978; Studium der Biologie an der Universität Bremen. Anfertigung der Diplomarbeit während eines Forschungsaufenthalts am Mental Health Research Institute, Melbourne, Australien. 2008 Promotion in Bremen. Schwerpunkt der wissenschaftlichen Arbeiten ist die Rolle des cannabinoiden Systems bei der Verhaltenssteuerung der Ratte und die Folgen einer chronischen pubertären Cannabinoidbehandlung.

Miriam Schneider: geboren 1976; Studium der Biologie an der Universität Tübingen. Promotion 2004 an der Universität Bremen. Wissenschaftliche Mitarbeiterin am Universitätsklinikum Köln. Seit 2006 wissenschaftliche Mitarbeiterin am Zentralinstitut für Seelische Gesundheit Mannheim. Forschungsschwerpunkt liegt auf Reifungsprozessen des endocannabinoiden Systems während der postnatalen Entwicklung (Pubertät) sowie auf der Beteiligung des endocannabinoiden Systems bei belohnungsrelevanten Prozessen.

Michael Koch: geboren 1959; Studium der Biologie und Chemie an der Universität Konstanz. Promotion 1990. Forschungsaufenthalte an der Universität Cambridge (UK). 1996 Habilitation (Tierphysiologie) in Tübingen. Heisenbergstipendiat bis 1999. Seit 2000 Professor für Neuropharmakologie an der Universität Bremen.

\section{Korrespondenzadresse}

\author{
Nico Wegener \\ Institut für Hirnforschung \\ Abteilung Neuropharmakologie \\ Universität Bremen / Cognium \\ 28395 Bremen$$
\text { Tel.: } \quad+49(0) 42121862978
$$ \\ E-Mail: nwegener@uni-bremen.de
}

\title{
Motorische Kontrolle der akustischen Orientierung von Grillen
}

\author{
Tom Baden, Maja Zorovic und Berthold Hedwig
}

Zusammenfassung

Das auditorische Orientierungsverhalten von Grillen ist eine komplexe senso-motorische Schleife, die von der Gesangswahrnehmung und Gesangserkennung bis hin zur Motorik der ausgelösten Steuerbewegungen reicht. Paarungsbereite Grillenweibchen laufen zu den singenden Männchen und benutzen deren Lockgesang als sensorischen Wegweiser. Dieses Verhalten, genannt Phonotaxis, ist auf des Zeitmuster des Männchengesangs abgestimmt. Doch während der Phonotaxis verändert sich die Selektivität des Verhaltens und die Tiere steuern reflexartig auch zu nichtattraktiven Lautpulsen. An den auditorischen Steuerbewegungen sind die Vorderbeine beteiligt, indem insbesondere die kontralaterale Tibia in Richtung der Schallquelle gestreckt wird. In den slow und fast Motoneuronen, die die Steuerbewegungen der Tibia kontrollieren, erhöht sich während rhythmischer Motoraktivität der zytosolische Kalziumspiegel in Phase mit den Motorsalven. Die Motoneurone erhalten keine direkten auditorischen Eingänge, sie zeigen aber bei elektrischer Reizung der Halskonnektive eine überschwellige synaptische Antwort. In laufenden Weibchen lösen identifizierte deszendierende Hirnneurone bei intrazellulärer Reizung starke Drehbewegungen aus und sind möglicherweise an der Richtungskontrolle des phonotaktischen Laufens beteiligt.

\section{Abstract}

Motor Control of Auditory Orientation in Crickets

Auditory orientation in female crickets involves a complex sensory-to-motor loop extending from song perception and pattern recognition to the motor control of orientation behaviour. Females, which are ready to mate, orient towards singing males using their calling song as a sensory cue. Their behaviour, called phonotaxis, is tuned towards the temporal pattern of the male song. However, during phonotaxis the selectivity of pattern recognition changes and the animals steer reflex-like to non-attractive sound pulses. Especially the front legs contribute to the auditory steering responses by extending the contralateral front tibia towards the sound source. During rhythmic motor activity the cytosolic $\mathrm{Ca}^{2+}$ levels in the slow-extensor and fast-flexor motoneurons that control the tibia movements increase in phase with motor bursts. The motoneurons do not receive any auditory inputs but they respond to electrical stimulation of the cervical connectives with suprathreshold synaptic activity. In walking crickets some identified interneurons descending from the brain evoke strong steering movements upon intracellular stimulation and thus may contribute to the control of phonotactic steering and walking.

Key words: phonotaxis; track ball system; reactive steering; motoneurons; calcium imaging

\section{Einleitung}

Grillen nutzen akustische Signale zur intra-spezifischen Kommunikation. Die Männchen erzeugen mit rhytmischen Bewegungen ihrer Vorderflügel einen artspezifischen Lockgesang. Mit jeder Schließbewegung der Flügel geht ein Lautpuls von ca. $20 \mathrm{~ms}$ Dauer $(4.8 \mathrm{kHz}$ bei ca. $100 \mathrm{~dB}$ SPL) einher. Mehrere dieser Pulse werden von den Tieren zu Versen gruppiert und ausdauernd vorgetragen, um Weibchen anzulocken (Huber 1964). Beide Geschlechter besitzen Hörorgane in den Vorderbeinen. Die Weibchen sind stumm, doch wenn sie paarungsbereit sind, laufen oder fliegen sie zu den singenden Männchen und nutzen dabei deren Gesang zur Orientierung (Popov und Shuvalov 1977; Ulagaraj und Walker 1973). Dieses Verhalten der Weibchen wird als Phonotaxis bezeichnet und stellt eine komplexe senso-motorische Schleife dar, die von der auditorischen Informationsverarbeitung bis hin zur Erzeugung 
der motorischen Steuerreaktionen reicht, die dem Orientierungsverhalten zugrunde liegen. Bisher ist insbesondere die auditorische Seite der Phonotaxis Forschungsgegenstand gewesen u.a. mit der Zielsetzung, die Richtungssensitivität des Systems und die neuronalen Filter zu verstehen, die die Erkennung des artspezifischen Gesangsmusters leisten (Hoy 1978; Schildberger et al. 1989; Lewis 1992; Pollack 2000). Die Ohren von Grillen liegen in den Vorderbeinen und arbeiten als Druckgradienten-Empfänger: Die Tympana sind den Schallwellen direkt ausgesetzt aber die Hörorgane erhalten den wesentlichen auditorischen Eingang über

werden die Reaktionen auf die Lautpulse schließlich in das motorische Lokomotionsmuster integriert? Geschieht dies über lokale thorakale Netzwerke oder durch deszendierende Hirnneurone? Nur wenig ist bekannt über die Beinbewegungen, die Motoneurone und die neuronalen Netzwerke, die das Gesangsmuster verarbeiten und das Laufverhalten kontrollieren. Wir gehen diesen Fragestellungen mit Verhaltensexperimenten nach, mit intrazellulären Ableitungen von Hirnneuronen in aktiv laufenden Weibchen und mit optischen Methoden, die die räumliche und zeitliche Dynamik von zytosolischen Kalziumände-

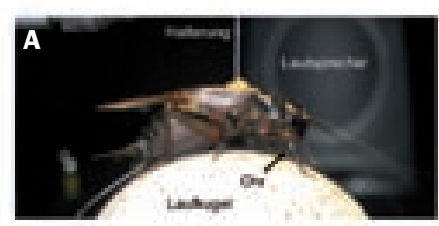

B
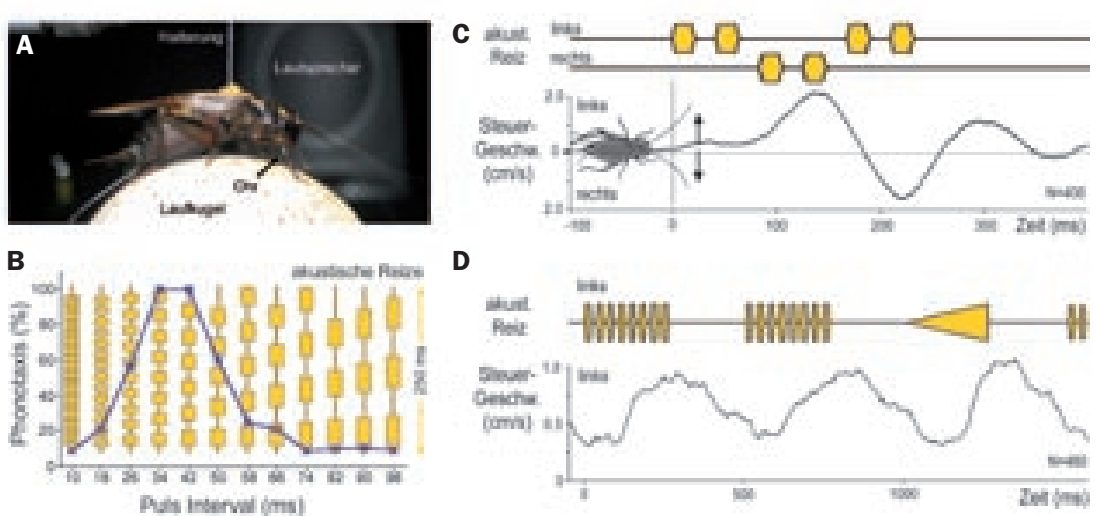

D

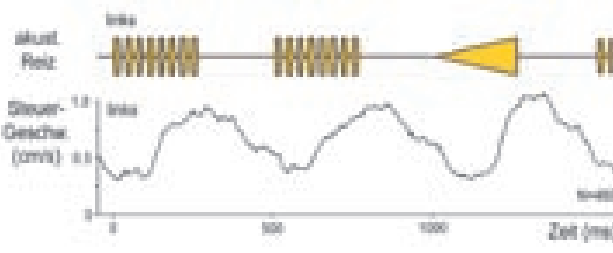

Abb. 1: (A) Ein Grillenweibchen, das an einer Halterung befestigt ist, läuft auf einer luftgelagerten Kugel während dem Tier akustische Reize über zwei Lautsprecher angeboten werden. Das Trommelfell im rechten Vorderbein ist deutlich zu erkennen. (B) Kennlinie der Phonotaxis bei 75 dB SPL. Die Weibchen zeigen eine deutliche Präferenz für Lautmuster mit eines Pulsrate von ca. $30 \mathrm{~Hz}$, die dem arteigenen Gesang entsprechen. Kürzere oder längere Pulse lösen keine Phonotaxis aus. (C) Die Weibchen reagieren mit schnellen Steuerbewegungen in Richtung der Lautpulse (Dauer 21 ms, Interval 21 ms), wenn diese paarweise aber abwechselnd von links und rechts angeboten werden. Richtungsänderungen erfolgen mit einer Latenz von 55-60 ms deutlich vor dem Ende des Vers. (D) Werden nichtattraktive Testpulse wie dieser Testreiz mit zunehmender Amplitude in das normale Gesangsmuster eingefügt, so steuern die Tiere während der Phonotaxis auch in Richtung dieser Testpulse. Reaktionen in C und D jeweils über 400 Reize gemittelt.

eine akustische Trachee, die hörrohrartig von der Körperwand des Thorax in das Vorderbein verläuft. Die linke und rechte akustische Trachee sind mechanisch miteinander gekoppelt und dieses Design verbessert die Richtungsempfindlichkeit des Systems (Lewis 1983; Michelsen und Larsen 2008). Ungefähr 50 primäre Sinneszellen reagieren auf akustische Reize und senden mit ihren Axonen ihre Spikemuster in das auditorische Neuropil im ersten thorakalen Ganglion. Nur zwei auditorische Neurone projizieren von hier in das Gehirn, wo anscheinend die Erkennung des arteigenen Gesangs (Boyan 1980; Schildberger 1984) und die Erzeugung der Steuerkommandos (Böhm und Schildberger 1992) für die auditorische Orientierung stattfindet. Doch wie rungen darstellen. Ziel dieser Arbeiten ist es, eine neuronale Verarbeitungsschleife, die einem komplexen Verhalten zugrunde liegt, von der Sensorik bis zur Motorik auf der zellulären und der Netzwerkebene umfassend zu verstehen.

\section{Verhaltensanalyse auf der Laufkugel}

Um die phonotaktische Orientierung der Weibchen zu analysieren, bieten wir den Tieren akustische Reizmuster an und messen gleichzeitig ihr Lauf- und Steuerverhalten. Die Grillen sind mit einer Halterung fixiert und stehen auf einer leichten aber stabilen Kunststoffkugel (Durchmesser 5.6 $\mathrm{cm}$, Masse $3 \mathrm{gr}$ ) (Abbildung 1A). Während des Laufens drehen sie die luftgelagerte
Kugel unter sich weg und ein optisches Sensorsystem misst die Rotation der Kugel um die Längs- und Querachse (i.e. vorwärtsrückwärts und links-rechts-Drehung) mit hoher zeitlicher und räumlicher Auflösung. Die Laufgeschwindigkeit und Laufrichtung der Weibchen können aus diesen Daten berechnet werden. (Hedwig und Poulet 2004, 2005). Das System erlaubt es, die phonotaktische Reaktion auf eine Palette von Reizmustern zu testen und so die Präferenzen der Weibchen zu bestimmen (Abbildung 1B). Das Verhalten der Weibchen ist eng auf das Zeitmuster des Lockgesangs der Männchen abgestimmt und ist besonders ausgeprägt, wenn den Tieren Lautpulse von ca. $20 \mathrm{~ms}$ Dauer mit einem Intervall von 15-20 ms (Wiederholrate 25-30 Hz; Intensität $75 \mathrm{~dB}$ SPL, Frequenz $4.8 \mathrm{kHz}$ ) angeboten werden. Kürzere und längere Lautpulse mit einer höheren bzw. niedrigeren Wiederholrate, lösen keine Phonotaxis aus. Dies hat zu der Hypothese geführt, dass die Gesangserkennung im Wesentlichen auf der Pulsrate des Gesangs basiert (Thorson und Weber 1982; Weber und Thorson 1989). Da die Tiere auf der Laufkugel unter open loop-Bedingungen laufen und ihre Position zur Schallquelle nicht ändern, sind auch detaillierte Analysen des Steuerverhaltens möglich, wenn z.B. die Richtung der akustischen Reizung geändert wird oder nichtattraktive Testreize in das Gesangsmuster eingefügt werden.

Eine zentrale Frage ist, für welche Zeitdauer oder Anzahl von Lautpulsen die Tiere das Gesangsmuster integrieren, bis sie eine Entscheidung über die Laufrichtung fällen? Frühe Experimente mit relativ trägen Laufkompensatoren zeigten Richtungsänderungen erst nach Wahrnehmung eines kompletten Gesangsverses und hatten vermuten lassen, dass die Tiere das arteigene Lautmuster erst erkennen müssen, bevor sie die Laufrichtung wechseln. Unsere Experimente mit Gesangsmustern, in denen aufeinanderfolgende Lautpulse im Wechsel von rechts oder links angeboten werden, zeigten, dass die Tiere reflexartige Steuerreaktionen zu einzelnen Lautpulsen durchführen (Abbildung 1C). Die Weibchen warten nicht $\mathrm{ab}$, bis sie einen vollständigen Vers gehört haben, sondern reagieren bereits nach dem ersten Lautpuls mit einer Latenz von 55-60 ms mit Änderungen ihrer Laufrichtung. Diese Zeit ist zu kurz, als dass die Mustererkennung direkt die Richtungsentscheidung beeinflussen könnte (Hedwig und Poulet 2004, 2005). Schließlich braucht ein Erkennungsystem mindestens zwei aufeinanderfolgende Lautpulse, um ihre Wiederholrate zu bestimmen. Anschei- 


\section{Spektrum Sachbücher Bildungsfutter und Lesespaß in einem!}

www.spektrum-verlag.de

\section{Das Wissen dieser Welt}

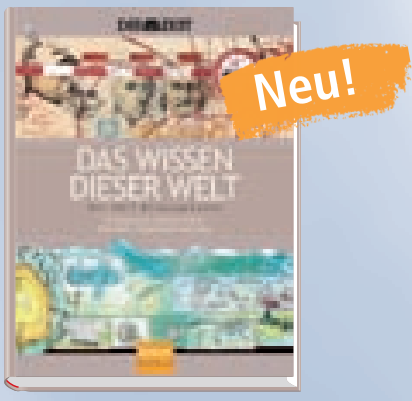

1. Aufl. 2008, 588 S., 100 Abb., geb.

$€$ (D) $49,95 / €$ (A) $51,35 /$ CHF 77,50

ISBN 978-3-8274-2089-3

\section{Die Zeit (Hrsg.)}

\section{Das Wissen dieser Welt}

Wie entsteht eine Demokratie? Wann wurde der Mensch zum Künstler? Wie arbeitet das Gehirn? In der größten und aufwändigsten Serie ihrer Geschichte hat die ZEIT 50 Wochen lang einen Kanon der wichtigsten Begriffe aus Politik und Wirtschaft, Natur- und Geisteswissenschaft, Kultur und Alltag aufgestellt.

\section{ZEIT-Redakteure sind um die} ganze Welt gereist - an historische Stätten, aktuelle Brennpunkte und an die Baustellen der Zukunft - um von dort aus die zentralen Ideen und Erkenntnisse mit Neugier, mit Leidenschaft und mit Kompetenz zu erforschen und darzustellen.

Entstanden sind 50 Reportagen, die das aktuelle Wissen unserer Zeit versammeln und Bildung auf einzigartige Weise lebendig gestalten.

\section{Der ZEIT-Bildungskanon}

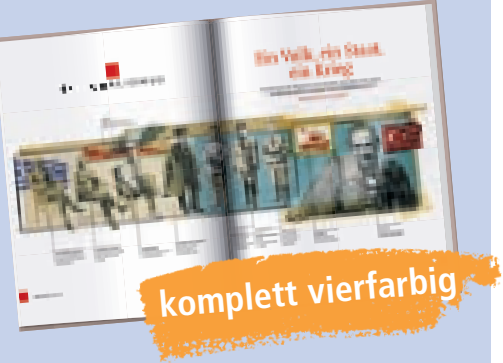

\section{Können Menschen biologisch} zum Bösen veranlagt sein?

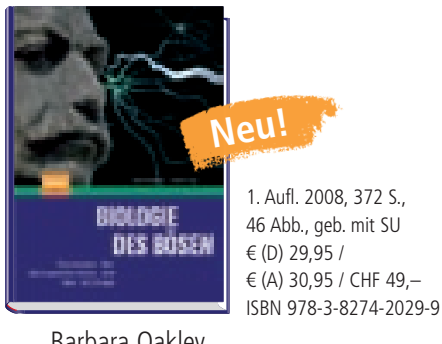

Barbara Oakley

Biologie des Bösen

Ausgehend von verstörenden Erfahrungen in der eigenen Familie deckt die Autorin Barbara Oakley auf, dass übel wollende Menschen oft aufgrund von körperlichen oder psychischen Fehlfunktionen so handeln. Sie setzt aktuelle psychologische, neurowissenschaftliche, verhaltensbiologische und genetische Entdeckungen in Beziehung zu den Charakteren vieler historischer Personen (allen voran Mao, Stalin, Hitler und Milosevic) und leitet daraus ein faszinierendes Bild vom Wirken des Bösen in der Politik, im Geschäftsund Sozialleben, in der Religion und sogar im privaten häuslichen Bereich ab.

\section{$>$ Wenn die Seele Trauer trägt}
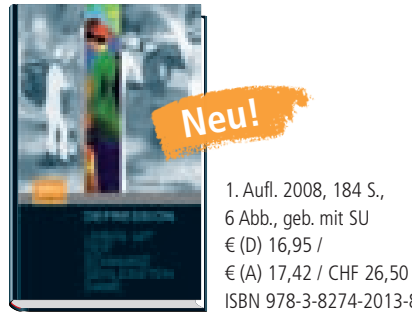

Thomas Haenel

\section{Depression}

„Die Depression kann mit einer in schwarz gekleideten Dame verglichen werden. Wenn sie kommt, so weise sie nicht weg, sondern bitte sie zu Tisch als Gast und höre, was sie Dir zu sagen hat.". ( C.G. Jung). Dieses Bild ist das Motto eines ungewöhnlichen Buches, in dem Thomas Haenel, über die vielen, teils noch unbekannten Gesichter der Depression allgemein verständlich berichtet. Haenels Buch lädt zum Zuhören ein und zeigt auf, wie sich Depressionen erkennen und behandeln lassen und wie man vorbeugen und Rückschläge bewältigen kann.

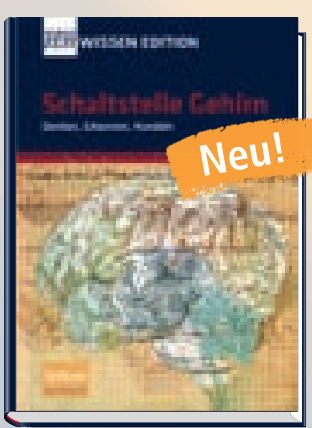

1. Aufl. $2008,294 \mathrm{~S}$,

100 Abb., geb.

$€(D) 24,95$ /

$€$ (A) $25,65 /$ CHF 41 ,

ISBN 978-3-8274-2002-2
Andreas Sentker / Frank Wigger (Hrsg.)

Schaltstelle Gehirn -

Werden wir das Gehirn je verstehen? Wissen wir, was wir denken? Können wir unser Fühlen Wollen und Handeln erklären, indem wir die chemischen und elektrischen Prozesse in den Nervenzellen aufzeichnen und analysieren? Dieser Band der "ZEIT WISSEN Edition" liefert einen schillernden Statusbericht von der neuround kognitionswissenschaftlichen Forschung. In den zahlreichen Beiträgen zeigen Psychologen, Hirnforscher und Biologen auf, was wir heute über die zentrale Schaltstelle Gehirn und ihre Aufgaben wissen - und welche Rolle sie für unser soziales Miteinander spielt. Den Beiträgen werden Reportagen, Analysen und Interviews namhafter ZEIT-Autoren zur Seite gestellt. Diese ordnen die wissenschaftlichen Positionen der Forscher in das Gesamtbild ein und zeigen Zusammenhänge und Widersprüche auf.
Denken, Erkennen, Handeln

\section{Warum gute Menschen böse werden ...} Vom weltbekannten Stanford Prison Experiment bis Abu Ghraib

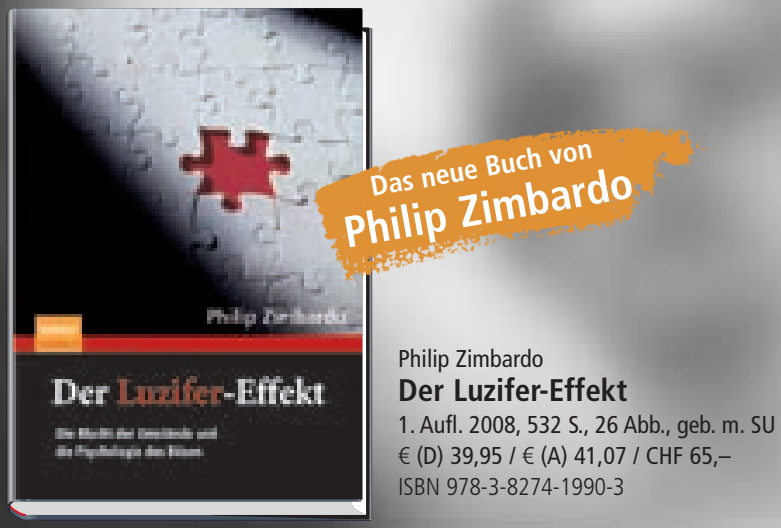

Was bringt gute Menschen dazu, Böses zu tun? Der renommierte Sozialpsychologe Philip Zimbardo, Leiter des weltbekannten Stanford Prison Experiment, erläutert in seinem neuen Buch Der Luzifer-Effekt, wie wir alle für die Versuchungen „der finsteren Seite" anfällig sind. Anhand historischer Beispiele sowie seiner eigenen bahnbrechenden Forschungen führt er detailliert aus, wie situative Kräfte und gruppendynamische Prozesse zusammenwirken können, um aus anständigen Männern und Frauen Ungeheuer werden zu lassen.

Eine schockierende und fesselnde Studie!

\section{Bequem bestellen:}

direkt bei www.spektrum-verlag.de

telefonisch: + $496221345-0$

per E-Mail: SDC-bookorder@springer.com per Fax: + $496221345-4229$ Der $€(A)$-Preis ist uns vom dortigen Importeur als Mindestpreis genannt worden. Der sFr-Preis ist eine unverbindliche Preisempfehlung. per Post: Springer Verlag Heidelberg

Kundenservice Bücher • Haberstrasse 7•D- 69126 Heidelberg 
nend reagieren die Tiere reflexartig auf die Lautpulse, wenn die Phonotaxis einmal ausgelöst ist. Die Laufrichtung wird nicht wie ein Vektor berechnet, sondern ergibt sich aus vielen aufeinanderfolgenden Steuerreaktionen. Die Reaktionen auf einzelne Lautpulse könnten so interpretiert werden, dass sich die Tiere wahllos akustischen Signalen hinwenden. Aber dies ist nicht der Fall, wie die Abstimmung der Phonotaxis auf das Zeitmuster des arteigenen Gesangs belegt. Wie ist dieser vermeintliche Widerspruch zu erklären?

Die Anpassung des phonotaktischen Verhaltens der Weibchen an das artspezifische Lautmuster der Männchen ist vielfach nachgewiesen worden (Weber und Thorson 1989) - aber tatsächlich ist diese Abstimmung dynamisch. Werden die Weibchen mit rampenförmig ansteigenden oder abfallenden Testpulsen von der Dauer eines Gesangsverses beschallt, so zeigen die
Tiere kein phonotaktisches Verhalten. Wir schließen daraus, dass diese Reize für die Tiere nicht attraktiv sind. Werden dieselben Testpulse jedoch in den normalen Lockgesang eingefügt, so steuern die Tiere auch auf diese rampenförmigen Laute und zwar mit einer Drehbewegung, die nicht kleiner ist als die Steuerbewegungen zu den normalen Gesangsversen (Abbildung 1D). Dasselbe gilt auch für andere Testreize, die allein keine phonotaktische Reaktion auslösen (Poulet und Hedwig 2005; Hedwig 2006). Die Reaktion auf solche nichtattraktiven Laute klingt innerhalb von ca. $5 \mathrm{~s}$ nach der Präsentation einer normalen Gesangssequenz vollständig ab. Sobald die Gesangserkennung aktiviert ist, vermindert sich also die Selektivität des auditorischen Systems und die Amplitude der Steuerbewegungen wird verstärkt. Die Erkennung eines Gesangsverses ist dann nicht mehr erforderlich, um eine Steuerreaktion auszulösen. Die Gesangserkennung

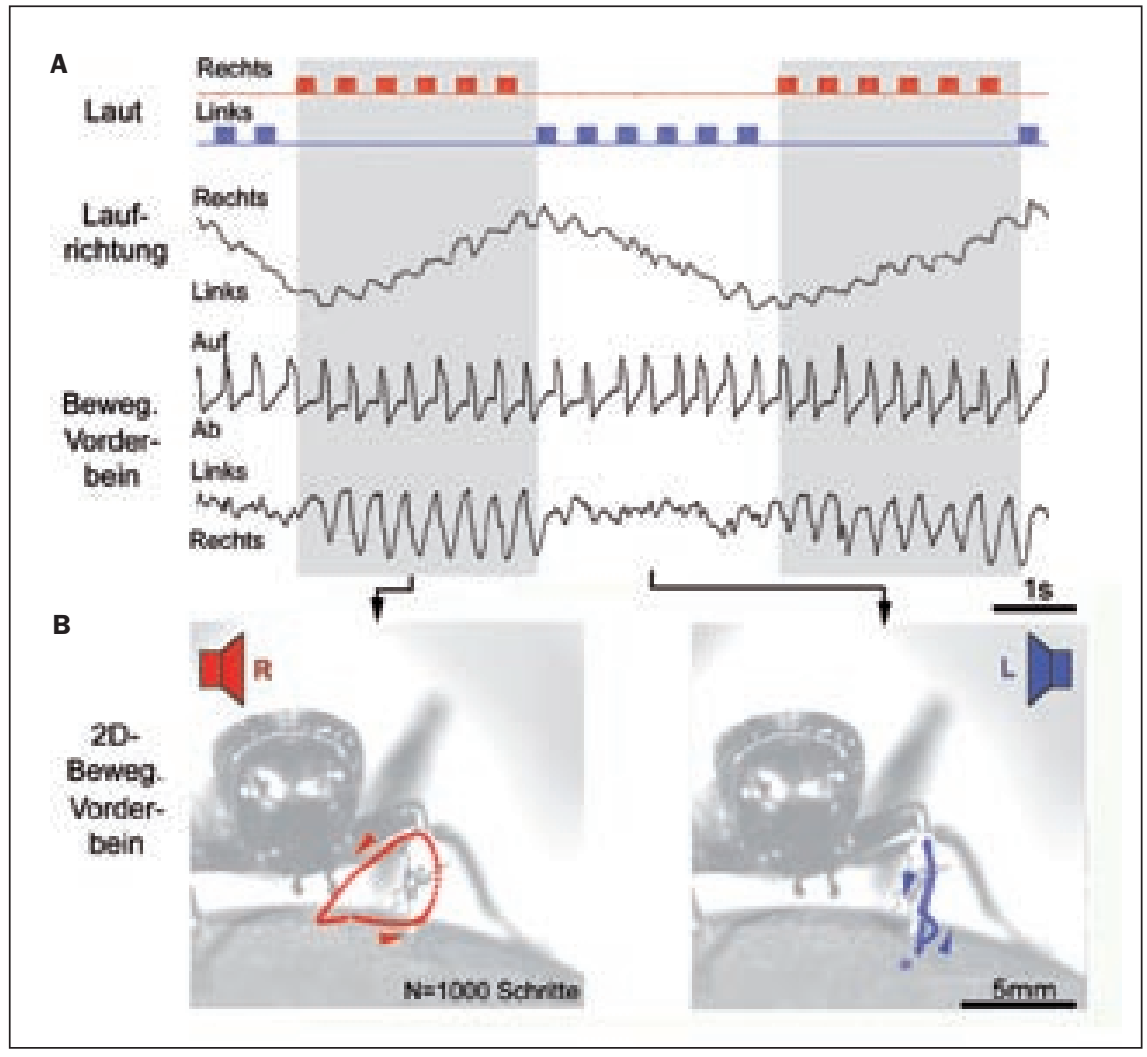

Abb. 2: (A) Bewegungen eines Vorderbeins während Phonotaxis. Das Weibchen steuert zu Gesangsversen, die im Wechsel von links und rechts dargeboten werden. Die auf-ab- und links-rechts- Bewegungen des linken Vorderbeins wurden gleichzeitig mit einer Photodiode gemessen. Während die auf-ab-Bewegunen unverändert bleiben, ändert das Tier die Seitwärtsbewegungen, sobald das akustische Reizmuster aus der neuen Richtung geboten wird. (B) Die Bewegungsspuren des Vorderbeines rekonstruiert von den gemittelten aufab- und links-rechts- Bewegungen. Zur Verdeutlichung sind die Bewegungsspuren auf die Vorderansicht einer Grille projiziert. Bei kontralateraler Reizung greift das linke Vorderbein weit nach rechts und zieht das Tier in Richtung der Schallquelle (B links). Bei ipsilateraler Reizung vollführt das Bein nur minimale seitliche Bewegungen (B rechts).

hat daher keinen direkten sondern nur einen indirekten Einfluss auf die Erzeugung der Steuerbewegungen. Der Vorteil dieser dynamischen Veränderungen der auditorischen Informationsverarbeitung ist, dass die Tiere kurzzeitig Änderungen des Lockgesangs tolerieren, die durch Störungen in der Schallausbreitung im Biotop hervorgerufen oder durch Unregelmäßigkeiten des Gesangsverhaltens bedingt sein können. Sobald das arteigene Gesangsmuster allerdings für einige Sekunden ausfällt, bricht diese Toleranz im Verhalten zusammen. Auf neuronaler Ebenen liegt diesen Änderungen der auditorischen Informationsverarbeitung möglicherweise eine Bahnung oder eine Modulation der senso-motorischen Schleife zugrunde.

\section{Vorderbeinbewegungen und Richtungsänderungen des phonotaktischen Laufens}

Was sind also die Ausgangsneurone dieser senso-motorischen Schleife, die das akustische Steuerverhalten kontrollieren? Den schnellen Änderungen der Laufrichtung, die mit der Laufkugel gemessen werden, müssen entsprechende Änderungen der Beinbewegungen zugrunde liegen, die Drehbewegungen in Insekten kontrollieren. Um diese zu erfassen, haben wir die Bewegungen eines Vorderbeins mit einer positionsempfindlichen Photodiode während der Phonotaxis gemessen. Hierfür wird eine kleine reflektierende Scheibe an die Tibia des Vorderbeins geklebt. Der Messpunkt wird frontal beleuchtet und das zurückfallende Licht von einer Photodiode mit zwei Messachsen erfasst, sodass die auf-ab- und die links-rechts- Bewegungen des Beines registriert werden können. Die Experimente zeigen, dass die auf-ab-Bewegungen der Vorderbeine durchweg sehr stereotyp durchgeführt werden und sich auch bei Änderung der Schallrichtung nicht wesentlich ändern (Fig. 2A, B). Im Gegensatz dazu werden die links-rechts-Bewegungen der Vorderbeine deutlich anders ausgeführt. Erfolgt die Schallreizung von der gleichen Seite zu dem gemessenen Vorderbein, so wird das Bein nach vorn gestreckt, aber nur selten ist eine Auslenkung des Beines in Richtung der Schallquelle zu beobachten. Erfolgt die akustische Reizung aber von der gegenüberliegenden Seite, so greift das Vorderbein in der Regel vor den Kopf weit nach kontralateral und zieht das Tier mit der nächsten Stemmphase in die Richtung der Schallquelle. Diese Beinbewegungen in Richtung kontralateraler Schallquelle gehen offensichtlich auf eine verstärkte Extension 
der Vorder-Tibia zurück. Möglicherweise sind zusätzlich auch Drehbewegungen der Coxa daran beteiligt. Diese beiden Bewegungskomponenten können von dem Messsystem aber nicht getrennt werden. Ähnlich wie bei der Stabheuschrecke belegen die Daten jedoch die Bedeutung der Vorderbeine für die Drehbewegungen der Tiere (Dürr und Ebeling 2005; Rosano und Webb 2007). Eine umfassende Analyse der Beinbewegung erfolgt nun mit einer Hochgeschwindigkeitskamera, die auch die Bewegung der Mittelund Hinterbeine messen kann.

\section{Identifizierung der Motoneurone}

Die Steuerbewegungen der Vorderbeine während der Phonotaxis weisen darauf hin, dass zumindest die Extensor- und Flexormuskeln der Tibia an diesen Bewegungen beteiligt sind. Wir haben deshalb die Aktivitätsmuster dieser Muskeln in laufenden Tieren untersucht (Baden und Hedwig 2008) und dazu feine $30 \mu \mathrm{m}$ durchmessende Stahldrähte in den Extensormuskel implantiert. Damit kann das Elektromyogram (EMG) des Extensors direkt und das des Flexors als Übersprechen mit kleiner Amplitude indirekt registriert werden. Wenn die Tiere laufen, zeigen diese Muskeln wie zu erwarten eine deutliche antagonistische Aktivierung im Laufrhythmus (Abbildung 3A). Die Aktivierung der Extensoren erfolgt in der Schwingphase und die Aktivierung der Flexoren in der Stemmphase der Beinbewegung. Ein Effekt der akustischen Reizung auf die Muskelaktivität ist in Einzelregistrierungen nicht offensichtlich aber wird dann deutlich, wenn die Muskelaktivität in Bezug auf die akustischen Reize gemittelt wird (Abbildung 3B). Der Extensormuskel der Tibia wird insbesondere bei ipsilateraler akustischer Reizung vermehrt eingesetzt und erhöht seine Aktivitätsrate von ca. $10 \mathrm{AP} / \mathrm{s}$ auf $15 \mathrm{AP} / \mathrm{s}$ in Phase mit den Lautpulsen. In entsprechender Weise wird der Flexormuskel bei kontralateraler Beschallung vermehrt aktiviert. In festgelegten Tieren konnten wir die Motoneurone dieser Muskeln identifizieren und ihre Verzweigungsmuster im ersten thorakalen Ganglion darstellen (Abbildung 3C). Während der Extensormuskel nur von einem schnellen (fast) und einem langsamen (slow) Motoneuron innerviert wird, sind an der Flexoraktivierung mindestens fünf fast und drei slow-Motoneurone beteiligt. Wie bei anderen Insekten ist die Flexorinnervierung deutlich komplexer als die des Extensors der Tibia (Burrows 1996). Von den Tibia-Motoneuronen werden insbesondere die fast-Flexoren und der slow-Extensor

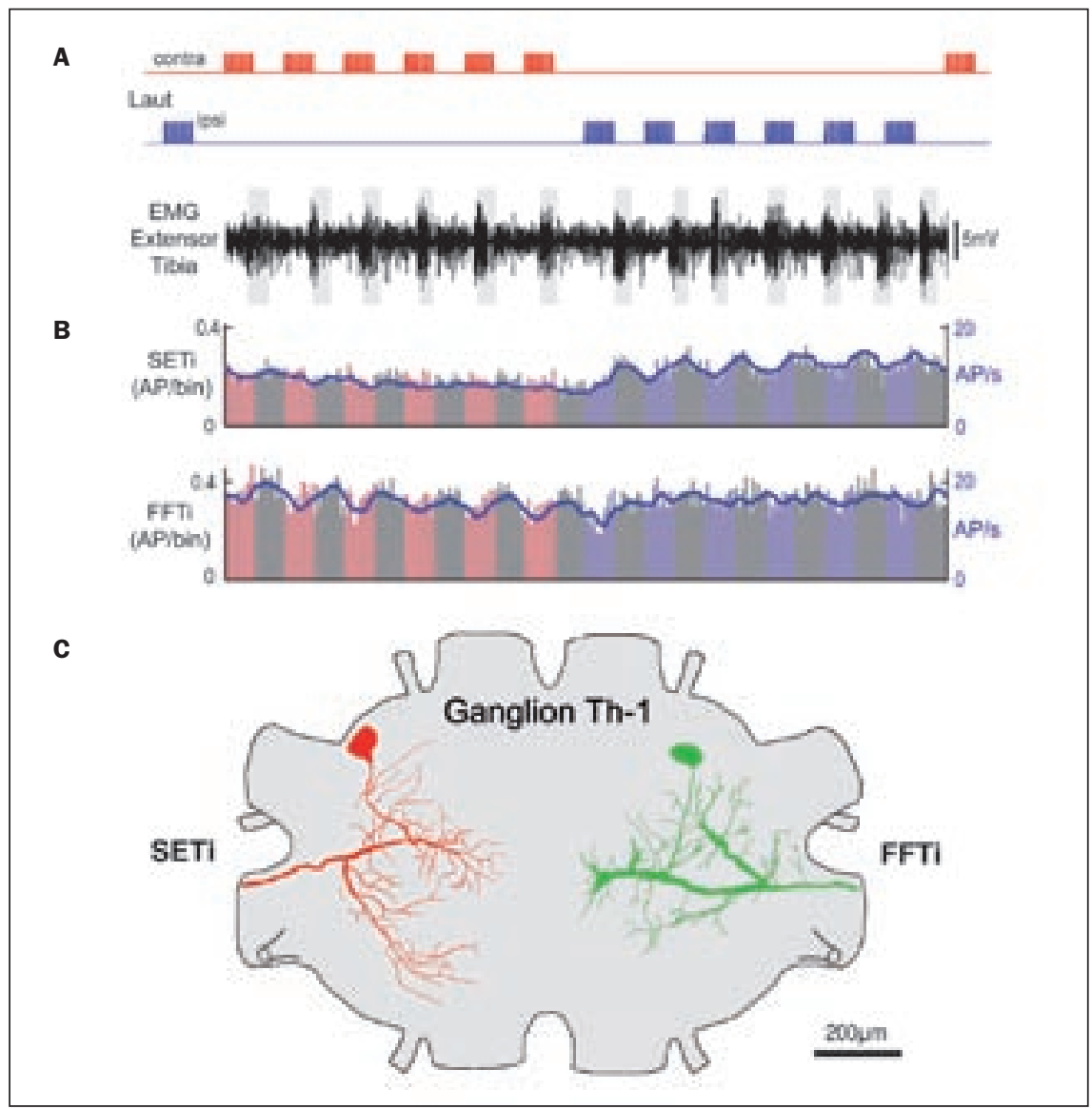

Abb. 3: (A) Ableitung des Elektromyograms des Extensormuskels der Vorderbein Tibia während eines phonotaktischen Laufes. Große EMG-Spikes zeigen die Aktiviät des Extensormuskels an und die kleinen Spikes zusätzlich die Aktivität des antagonistischen Flexormuskels. Das Gesangsmuster wird in einer Folge von links und rechts angeboten. In der Ableitung maskiert die Modulation der Muskelaktivität im Laufrhythmus die Reaktion auf die akustischen Reize. (B) Eine quantitative Analyse der Muskelaktiviät mit PST-Histogramm (linke Achse) und Spikefrequenz (rechte Achse) zeigt eine rhythmische Modulation des Extensors in Phase mit ipsilateraler akustischer Reizung und eine rhythmische Modulation der Flexoraktivität bei kontralateraler Reizung. (C) Struktur des slow Extensor-Tibia-Motoneurons (SETi) und eines fast Flexor-Tibia-Motoneurons (FFTi) im ersten thorakalen Ganglion.

während der Phonotaxis aktiviert. Die dendritischen Verzweigungen der Neurone liegen im dorsalen Neuropil des ersten thorakalen Ganglion und sind damit deutlich außerhalb des ventralen auditorischen Neuropils. Lokale auditorische Eingänge, die die Grundlage eines direkten Reflexes von den auditorischen Neuronen zu den Motoneuronen bilden könnten, sind somit ausgeschlossen. Weiterhin reagieren die tibialen Motoneurone in festgelegten Tieren auch nicht unterschwellig auf akustische Reize. Die Motoneurone erhalten allerdings massive erregende polysynaptische Eingänge bei Reizung der anterioren Konnektive, die das thorakale Ganglion mit dem Gehirn verbinden. Eine effiziente erregende Ansteuerung der Motoneurone durch das Gehirn ist somit zumindest möglich. Die
Kenntnis der synaptischen Aktivierung der Motoneurone während der Phonotaxis würde interessante Rückschlüsse auf die auditorische Informationsverarbeitung erlauben, doch sind intrazelluläre Ableitungen von Motoneuronen in laufenden Grillen technisch extrem schwierig. Wir haben daher die zellulären Eigenschaften dieser Neurone in festgelegten Tieren näher untersucht.

\section{Optische Darstellung von Kalzium- signalen in tibialen Motoneuronen}

Kalziumionen spielen in Neuronen eine zentrale Rolle u.a. bei der synaptischen Übertragung, der Aktivierung von Ionenkanälen oder der Regulierung von Genaktivität (Berridge 1998; Bootman et al. 2001; 


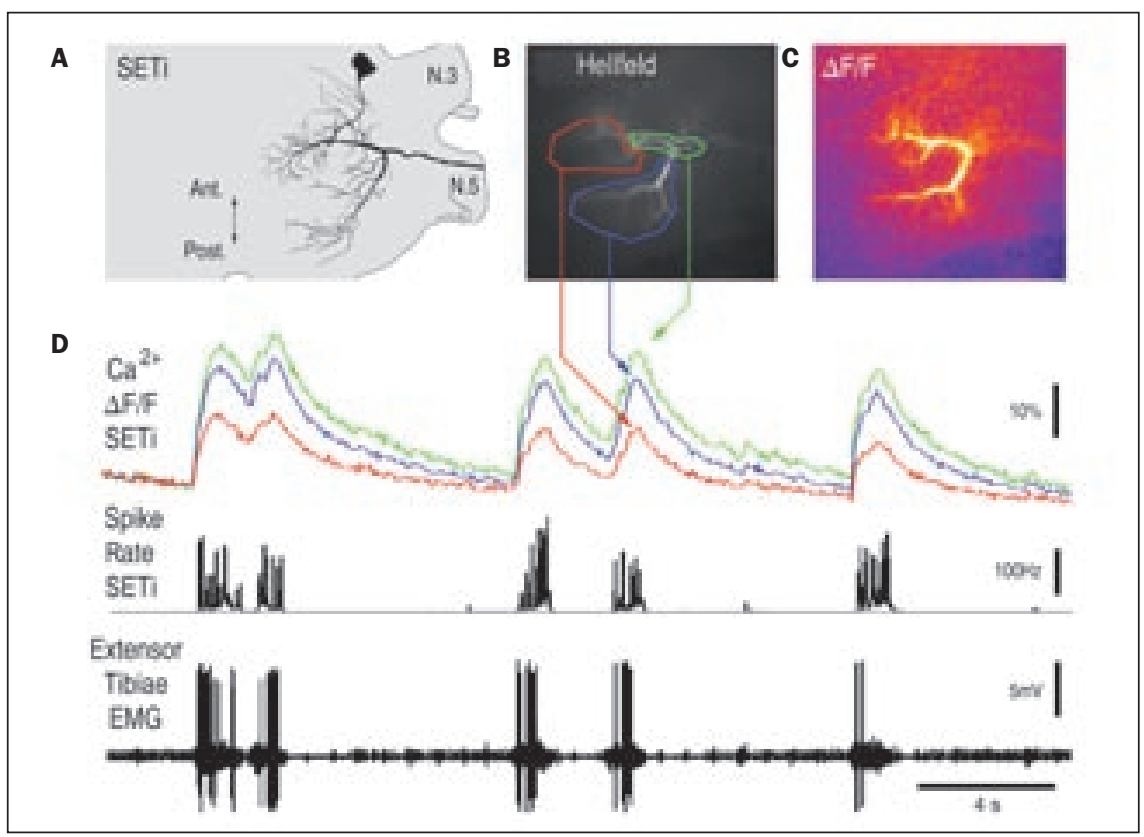

Abb. 4: (A-C) Relative Änderungen der zytosolischen Kalziumkonzentration im slow Extensor-Motoneuron (SETi) während rhythmischer motorischer Aktivität. Sowohl im Hellfeld als auch bei der Fluoreszenzmessung können die primären und die sekundären Neuriten optisch deutlich aufgelöst werden. Das Kalziumsignal ist für drei Regionen der Zelle bestimmt worden. (D) Mit jeder motorischen Salve des Extensormuskels erhöht sich die Konzentration des zytosolischen Kalziums. Diese Änderungen klingen nur langsam ab. Das Elektromyogramm des Extensormuskels wurde genutzt, um die Spikeaktivität des SETi-Motoneurons zu bestimmen.

Augustine et al. 2003). Das Ion ist dadurch ein geeigneter Indikator für neuronale Aktiviät. Farbstoffe, die an Kalzium binden und dabei ihre Fluoreszenz ändern, erlauben es, die räumliche und zeitliche Verteilung des zytosolischen Kalziums darzustellen und in ruhenden und aktivierten Neuronen in vivo zu vergleichen. Die Dendriten der tibialen Motoneurone liegen ca. $200 \mu \mathrm{m}$ tief im ersten thorakalen Ganglion und erstrecken sich parallel zu dessen dorsaler Oberfläche. Diese strukturellen Voraussetzungen sind günstig, um die Kalziumänderungen in den Eingangsverzweigungen der Neurone mit optischen Methoden zu analysieren. Dazu haben wir Farbstoffe wie Oregon Green BAPTA-1 in die Motoneurone mit scharfen Mikroelektroden iontophoretisch injiziert und die Fluoreszenzänderungen des Farbstoffs gemeinsam mit der elektrischen Aktivität der Neurone während rhytmischer motorischer Aktiviät analysiert (Abbildung 4A-D). Der Motorrhythmus wurde durch systemische Applikation von Pilocarpin ausgelöst und das Elektromyogramm (EMG) des Muskels abgeleitet, um die Spikeaktivität des Extensors zu erfassen. Zur Messung der Fluoreszenz diente eine gekühlte CCD - Kamera mit einem 256 x 256 Pixel großen optischen Sensor und einer Videorate von $50 \mathrm{~Hz}$. Unsere Aufnahmen zeigen deutliche Änderungen des Fluoreszenzsignals im Rhythmus der Extensoraktivität, die Entladungsraten bis zu $100 \mathrm{~Hz}$ erreicht. Kalziumänderungen können deutlich in den primären und den sekunären Dendriten optisch aufgelöst werden und zeigen für verschiedenen Regionen (Abbildung 4B) eine sehr ähnliche Dynamik (Abbildung 4C, D). Mit jeder Motorsalve erfolgt eine schnelle Zunahme ( $\tau \sim 150-200$ ms) des Kalziumsignals und anschließend eine sehr graduelle, deutlich langsamere Abnahme des Signals ( $\tau \sim 1000 \mathrm{~ms})$. Erfolgen die motorischen Salven in schneller Abfolge wie z.B. beim Laufen, so ergibt sich eine dauerhafte Erhöhung des Kalziumspiegels in der Zelle, während sie bei episodischer Aktivierung mit niedrigem Kalziumspiegel arbeiten. Ob sich daraus funktionelle Konsequenzen für die Aktivierung der Motoneurone, die Signalverarbeitung und die motorische Kontrolle des Laufverhalten ergeben, ist noch nicht vorherzusehen. In auditorischen Neuronen von Grillen löst das Kalzium einen Auswärtsstrom aus, der die Zellen hyperpolarisiert und Antworten auf akustische Reize mit niedriger Amplitude unterdrückt (Sobel und Tank 1994; Baden und Hedwig 2007).

\section{Kontrolle des Laufverhaltens durch Hirnneurone}

Von zentraler Bedeutung ist die Verarbeitung der auditorischen Signale im Gehirn der Tiere. Nur zwei aufsteigende auditorische Neurone ziehen vom auditorischen Neuropil im ersten thorakalen Ganglion in das Protocerebrum, wo die Erkennung des Gesangsmusters und die Kontrolle des phonotaktischen Verhaltens stattfindet. Im Gehirn gibt es kein offensichtlich abgegrenztes Neuropilgebiet, das ausschließlich der Verarbeitung auditorischer Reize zugewiesen wäre. Lokale Hirnneurone, die auditorische Reizmuster in unterschiedlicher Weise abbilden, haben weitläufige Verzweigungsmuster im protocerebralen Neuropil (Boyan 1980; Schildberger 1984). Um einen Zugang zur Gesangserkennung und die Generierung der Steuerkommandos zu erhalten, registrieren wir die Aktivität einzelner Hirnneurone, während die Tiere auf der Kugel laufen und auditorische Reizmuster präsentiert werden (Abbildung 5A). Wir erwarten, dass nur in solchen Tieren, die tatsächlich Phonotaxis zeigen, die entscheidenden auditorischen Netzwerke auch funktionell aktiviert sind. Für diese Experimente wird die Kopfkapsel festgelegt und einzelne Hirnneurone werden mit scharfen Mikroelektroden abgeleitet. Die Aktivitätsmuster der Neurone können so in stehenden und in laufenden Tieren registriert werden. Wird durch intrazelluläre Strominjektion die Spikeaktivität der Neurone moduliert, so kann geprüft werden, ob dies Änderungen im Verhalten der Tiere zur Folge hat und es erlaubt die funktionelle Bedeutung der Neurone zu beschreiben. Wir konnten mehrere Interneurone identifizieren, die an der Kontrolle des Laufens beteiligt sind oder während des Laufens aktiviert werden (Böhm und Schildberger 1992; Staudacher 2001). Das Interneuron in Abbildung 5B hat Dendriten hauptsächlich im posterioren Proto- und Deutocerebrum. Im Tritocerebrum haben die Verzweigungen die Erscheinung von axonalen Kollateralen, sodass hier bereits eine Ausgangsregion des Neurons vorliegt. In Bezug zum Zellörper verläuft das Axon im ipsilateralen Konnektiv.

Das Neuron war nicht aktiv in stehenden Tieren, aber Spikes traten auf sobald das Tier spontan anfing zu laufen. Akustische Reize hatten keinen Effekt auf die Neuronaktiviät. Wenn das Interneuron mit $2.5 \mathrm{nA}$ depolarisiert wurde, erhöhte sich die Spikerate auf ca. $200 \mathrm{~Hz}$ und die Grille steuerte deutlich zur Seite des absteigenden Axons, solange die Neuronäktiviät durch den Strompuls erhöht wurde (Abbildung 5C). Die Steuerbewegung folgte in ihrer Amplitude deutlich 
dem Verlauf der erhöhten Spikeaktivität. Die Aktivität des Interneurons war also hinreichend, um Steuerbewegungen auszulösen aber nicht notwendig, da Drehbewegungen auch auftraten, wenn das Neuron hyperpolarisiert wurde. Dieses Interneuron kann daher Teil eines Netzwerkes sein, das die motorischen Kommandos für die Phonotaxis an die thorakalen Ganglien weiterleitet. Der entscheidende Test steht jedoch noch aus, d.i. diese Neurone während phonotaktischen Verhaltens abzuleiten, um zu zeigen dass sie tatsächlich durch auditorische Reize angetrieben werden.

\section{Ausblick}

Das phonotaktische Verhalten von Grillen umfasst eine senso-motorische Schleife, in der artspezifische Kommunikationssignale erkannt werden und ein gerichtetetes Laufverhalten auslösen. Wir haben mittlerweile eine gute Kenntniss der auditorischen Bahn und beginnen die motorische Kontrolle des Laufverhaltens und der Steuerbewegungen zu verstehen. Nun kommt es darauf an, die zentrale und entscheidende Verbindung zwischen der Eingangs- und den Ausgangsneuronen des phonotaktischen Netzwerkes im Gehirn aufzuklären.

\section{Literatur}

Hedwig, B. und Poulet, J.F.A. (2004): Complex auditory behaviour emerges from simple reactive steering. Nature 430: 781-785.

Hedwig, B. und Poulet, J.F.A. (2005): Mechanisms underlying phonotactic steering in the cricket Gryllus bimaculatus (de Geer) revealed with a fast trackball system. J Exp Biol 208: 915-927.

Poulet, J.F.A. und Hedwig, B. (2005): Auditory orientation in crickets: Pattern recognition controls reactive steering. PNAS 102: 4717-4725.

Hedwig, B. (2006): Pulses, patterns and paths: neurobiology of acoustic behaviour in crickets. J Comp Physiol A 192: 677-689.

Baden, T. und Hedwig, B. (2007): Neurite specific $\mathrm{Ca}^{2+}$ dynamics underlying sound processing in an auditory interneurone. $J$ Neurobiol 67 : 68-80.

Baden, T. und Hedwig, B. (2008): Front Leg Movements and Tibial Motoneurons underlying auditory steering in the cricket (Gryllus bimaculatus de Geer). J Exp Biol 211: 2123-2133.

Eine vollständige Literaturliste kann bei den Autoren angefordert werden.

\section{Danksagung}

Die Arbeiten wurden unterstützt vom BBSRC, der Royal Society, dem Newton Trust Cambridge und dem Cambridge European

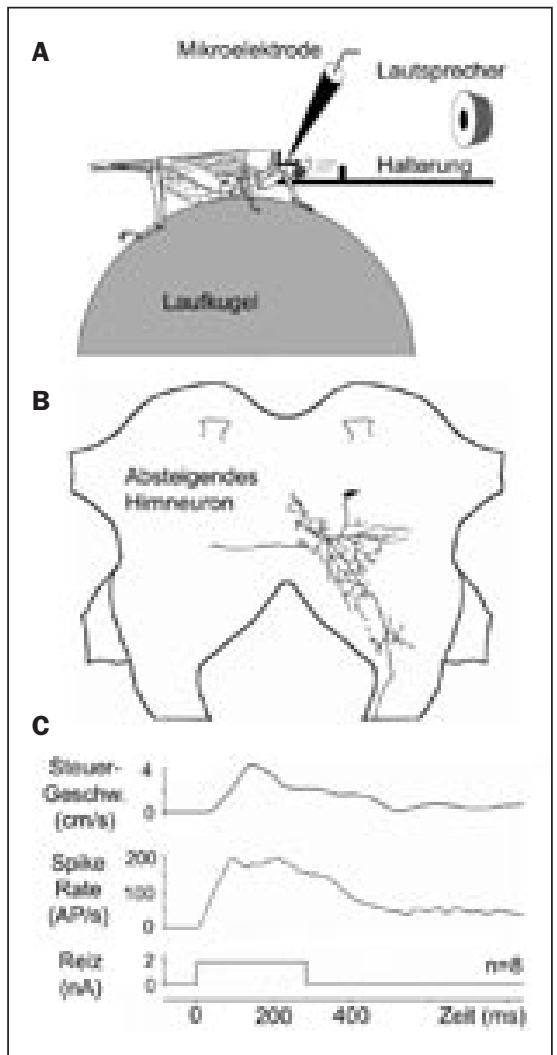

Abb. 5: (A) Versuchsaufbau zur Messung der Aktivität von Hirnneuronen in laufenden Grillen. (B) Ein Hirnneuron mit einem absteigenden Axon und dendritischen Verzweigungen im dorsalen Hirnbereich. (C) Aktivierung des Neurons mit einem depolarisierenden Strompuls erhöht die Aktivität des Neurons auf ca 150 AP/s und löst gleichzeitig eine deutliche Drehbewegung zu der Seite aus, auf der das Axon verläuft.

Trust. M. Knepper danken wir für die Weiterentwicklung von NEUROLAB.

\section{Kurzbiografien}

Dr. Berthold Hedwig: 1974 bis 1979 Biologie-Studium an der Universität Köln. Doktorarbeit am I. Zoologischen Institut der Universität Göttingen von 1980-1985. Danach Akademischer Rat und Hochschulasisstent an der Universität Göttingen und Habilitation im Jahr 1993. Von 1994-1997 Heisenberg-Stipendiat der DFG mit Forschungsaufenthalten bei H. Römer/Graz und M. Burrows/Cambridge. Ab 1997 Lecturer und nun Reader in Neuroscience am Department of Zoology/Cambridge.

Dr. Maja Zorovic: Studium der Biologie an der Universität Ljubljana/Slovenien. Diplomarbeit in der Abteilung Zoologie und Ökotoxikologie. Magister und Doktorarbeit am Institut für Entomologie des Nationalen Instituts für Biologie in Ljubljana unter Leitung von Prof. Dr. Andrej Čokl. Seit 2005 Postdoc am Department of Zoology/ Cambridge in der Arbeitsgruppe von B. Hedwig.

Dr. Tom Baden: Von 2001-2004 Studium der Naturwissenschaften und Neurowissenschaften an der Universität Cambridge. Seit 2004 Doktorand in der Arbeitsgruppe von B. Hedwig mit erfolgreichem Abschluss im April 2008. Er ist nun Postdoc im Labor von Dr. Leon Lagnado am MRC-LMB in Cambridge.

\section{Korrespondenzadresse}

\section{Dr. Berthold Hedwig}

University of Cambridge, Dept. of Zoology Downing Street, Cambridge, CB2 3EJ, UK

Tel.: $\quad+44(0) 1223336651$

Fax: $\quad+44(0) 1223330934$

E-Mail:bh202@cam.ac.uk

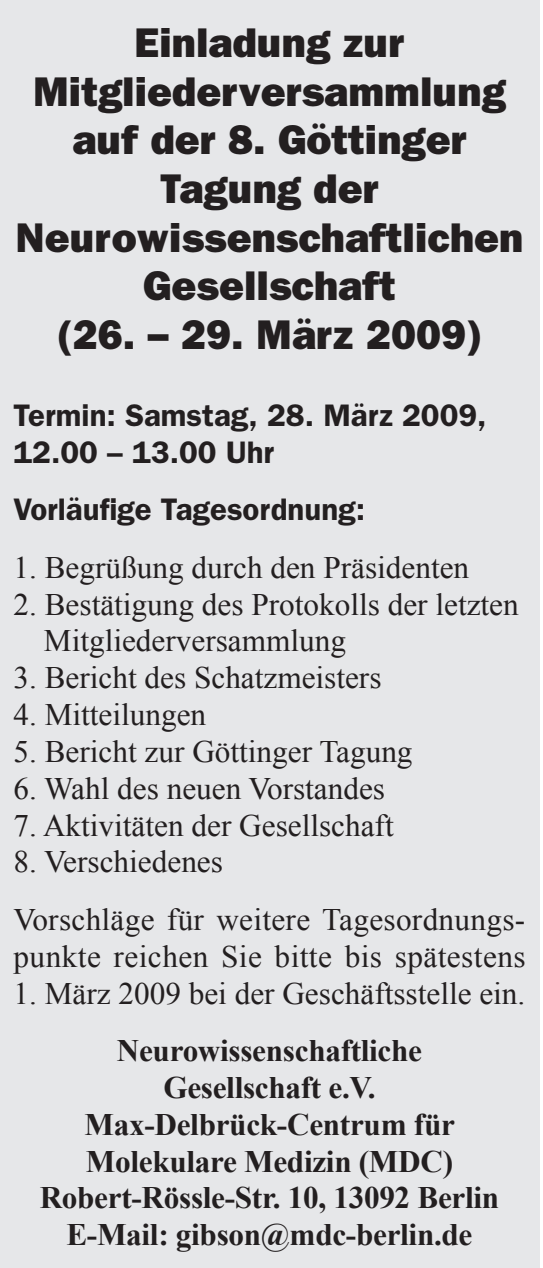

